\title{
Heavy quark form factors at three loops
}

\author{
Johannes Blümlein \\ Deutsches Elektronen-Synchrotron, DESY 15738 Zeuthen, Germany \\ E-mail: johannes.bluemlein@desy.de
}

\section{Peter Marquard*}

Deutsches Elektronen-Synchrotron, DESY 15738 Zeuthen, Germany

E-mail: peter.marquardedesy.de

\section{Narayan Rana}

INFN, Sezione di Milano, Via Celoria 16, I-20133 Milano, Italy

E-mail: narayan.rana@mi.infn.it

\section{Carsten Schneider}

Johannes Kepler University Linz, Research Institute for Symbolic Computation (RISC),

Altenbergerstraße 69, A-4040, Linz, Austria

E-mail: cschneiderisc.jku.at

We present results for the heavy-fermionic corrections to the non-singlet heavy-quark form factors at three-loop order recently obtained in [1].

14th International Symposium on Radiative Corrections (RADCOR2019)

9-13 September 2019

Palais des Papes, Avignon, France

* Speaker.

${ }^{\dagger}$ DESY 19-219, DO-TH 19/14, SAGEX-19-32 


\section{Introduction}

The knowledge of the massive three-loop form factor is an essential ingredient to the calculation for a series of massive processes at $e^{+} e^{-}$and hadron colliders, determined by vector, axialvector, scalar and pseudoscalar currents. It has been calculated to two-loop order in Refs. [2-7]. At three-loop order the color planar contributions have been computed in Refs. [8-13] and its asymptotic behaviour has been studied in $[14,15]$, including partial results at four-loop order.

In this proceedings, we review the computation of the non-singlet heavy-fermionic contributions of the massive three-loop form factor for vector, axialvector, scalar and pseudoscalar currents recently obtained in [1].

The basic structure of the massive form factors has been described in Ref. [7] before. We consider vector, axialvector, scalar and pseudoscalar currents coupling to a heavy quark pair of mass $m$

$$
\bar{u}_{c}\left(q_{1}\right) X_{c d} v_{d}\left(q_{2}\right)
$$

with $q=q_{1}+q_{2}$. The main variable considered is $x$ given by

$$
\frac{q^{2}}{m^{2}}=z=-\frac{(1-x)^{2}}{x}
$$

We work in $D=4-2 \varepsilon$ dimensions. In the axialvector and pseudoscalar case we can use an anticommuting $\gamma_{5}$ since we only consider the non-singlet contributions.

We consider the decay amplitude $\left(\Gamma^{\mu}\right)$ of the $Z$-boson into a pair of heavy quarks. The general structure of $\Gamma^{\mu}$ consists of six form factors, two of which are CP odd. As we consider only higher order QCD effects and Standard Model (SM) neutral current interactions to lowest order, the CP invariance holds. This implies that $\Gamma^{\mu}$ has four form factors $F_{V, i}(s), F_{A, i}(s) i=1,2$ comprising the following general form

$$
\begin{aligned}
\Gamma_{c d}^{\mu} & =\Gamma_{V, c d}^{\mu}+\Gamma_{A, c d}^{\mu} \\
& =-i \delta_{c d}\left[v_{Q}\left(\gamma^{\mu} F_{V, 1}+\frac{i}{2 m} \sigma^{\mu v} q_{v} F_{V, 2}\right)+a_{Q}\left(\gamma^{\mu} \gamma_{5} F_{A, 1}+\frac{1}{2 m} q^{\mu} \gamma_{5} F_{A, 2}\right)\right]
\end{aligned}
$$

where $\sigma^{\mu \nu}=\frac{i}{2}\left[\gamma^{\mu}, \gamma^{v}\right], q=q_{1}+q_{2}$, and $v_{Q}$ and $a_{Q}$ are the SM vector and axial-vector coupling constants as defined by

$$
v_{Q}=\frac{e}{\sin \theta_{w} \cos \theta_{w}}\left(\frac{T_{3}^{Q}}{2}-\sin ^{2} \theta_{w} Q_{Q}\right), \quad a_{Q}=-\frac{e}{\sin \theta_{w} \cos \theta_{w}} \frac{T_{3}^{Q}}{2} .
$$

$e$ is the charge of positron, $\theta_{w}$ is the weak mixing angle, $T_{3}^{Q}$ is the third component of the weak isospin, and $Q_{Q}$ is the charge of the heavy quark.

Furthermore, we consider a general neutral particle $h$ that couples to heavy quarks through the following Yukawa interaction

$$
\mathscr{L}_{i n t}=-\frac{m}{v}\left[s_{Q} \bar{Q} Q+i p_{Q} \bar{Q} \gamma_{5} Q\right] h,
$$


where $m$ denotes the heavy quark mass, $v=\left(\sqrt{2} G_{F}\right)^{-1 / 2}$ is the SM Higgs vacuum expectation value, with $G_{F}$ being the Fermi constant, $s_{Q}$ and $p_{Q}$ are the scalar/pseudoscalar coupling, respectively, and $Q$ and $h$ are the heavy quark and scalar and pseudoscalar field, respectively. The decay amplitude of $h \rightarrow \bar{Q}+Q, X_{c d} \equiv \Gamma_{c d}$, consists of two form factors with the following general structure

$$
\begin{aligned}
\Gamma_{c d} & =\Gamma_{S, c d}+\Gamma_{P, c d} \\
& =-\frac{m}{v} \delta_{c d}\left[s_{Q} F_{S}+i p_{Q} \gamma_{5} F_{P}\right],
\end{aligned}
$$

where $F_{S}$ and $F_{P}$ denote the renormalized scalar and pseudoscalar form factors, respectively. The form factors obey the expansion

$$
F_{i, l}\left(x, a_{s}\right)=\delta_{l, 1}+\sum_{k=1}^{\infty} a_{s}^{k} F_{i}^{(k)}(x),
$$

with $i=V, A, S, P$ and $l=1,2$ for $i=A, V$ and $a_{s}=\alpha_{s} /(4 \pi)$ denotes the strong coupling constant.

\section{Calculation and Results}

To calculate the form factors for the various currents we follow to a large extend the commonly used approach. The diagrams are generated using ggraf [16], the resulting diagrams are mapped on prototypes using $q 2 \mathrm{e} / \exp [17,18]$, projectors are applied and the expressions are simplified using (T) FORM $[19,20]$. The resulting scalar integrals are reduced to a small set of master integrals using integration-by-parts identities [21] implemented in the program Crusher [22]. This procedure allows to represent the form factors as a linear combination of 103 master integrals, which still have to be calculated. The calculation of the individual master integrals is a difficult task since they involve elliptic or even more complicated structures. Therefore, we continue using the method of arbitrarily large moments [23]. Compared to the complete analytic representation their expansion about $x=1$ can be fairly easily calculated. Therefore we first calculate the master integrals in an expansion about $x=1$. To obtain this expansion we insert a suitable ansatz into the coupled system of differential equations for the master integrals thus obtaining a system of coupled linear equations for the expansion coefficient. From these linear equations we can either calculate a (small) number of expansion coefficients by directly solving the system of equations. Likewise one may deduce recursions using SolveCoupledSystems [23] which allow for the calculation of arbitrary many. Having obtained the expansions for the master integrals we can now calculate the corresponding expansion of the full expressions for the form factors. In the final step, working under the assumption that the sum is simpler then the parts, we try to obtain a closed analytic form for the form factors by summing the obtained power series. To achieve this, we first determine a recurrence using guessing [24] for the different parts corresponding to the constants introduced by the boundary conditions. In the case that the recurrence is first-order factorizing it can be solved using Sigma [25-27], i.e. an explicit representation of the $n$-th expansion coefficient can be given. Once this representation is available it is possible to sum up the power series and find a closed analytic solution. The resulting infinite sums were then converted into harmonic polylogarithms using the package HarmonicSums [28-36]. In the case of the first-order factorizable recurrences the 
corresponding solutions are in general given by iterated integrals, cf. [37]. Here, we encounter only harmonic polylogarithms (HPLs) [38]. We needed at most 8000 moments to obtain the recursions for the various parts of the form factors. For details of the method and its implemenation we refer the reader to [1].

Let us illustrate the procedure with an example:

- We start with the sequence for $C_{i}$ in $\sum C_{i} y^{i}$

$$
\begin{array}{r}
\left\{-2,0,-\frac{1}{6},-\frac{1}{6},-\frac{3}{20},-\frac{2}{15},-\frac{5}{42},-\frac{3}{28},-\frac{7}{72},-\frac{4}{45},-\frac{9}{110},-\frac{5}{66},-\frac{11}{156},-\frac{6}{91},\right. \\
\left.-\frac{13}{210},-\frac{7}{120},-\frac{15}{272},-\frac{8}{153},-\frac{17}{342},-\frac{9}{190},-\frac{19}{420}, \ldots\right\}
\end{array}
$$

- guess the recurrence

$$
n^{2} C_{n}-(n-1)(n+2) C_{n+1}=0
$$

- find a solution for the recurrence

$$
C_{n}=\frac{1-n}{n(n+1)}
$$

which in this case is valid starting from $n=1$

- finally, performing the sum over $n=1, \ldots, \infty$ and adding the constant term $C_{0}$ yields

$$
-2+\sum_{n=1}^{\infty} \frac{1-n}{n(n+1)} y^{n}=-\frac{(y-2) \log (1-y)}{y} \stackrel{y \rightarrow 1-x}{=} \frac{(1+x) \log (x)}{1-x} .
$$

For many parts including all pole contributions we have been able to obtain closed analytic results. In Table 1 we summarize the properties of the recurrences which are not first-order factorizing and thus we could not solve at this point.

The analytic results are too lengthy to be reproduced here in closed form and we refer the reader to [1], where all results have been presented. For illustration we show in Fig. 1 the results for the vector form factor $F_{V, 1}$ in the regions $x \in[0,1]$, which corresponds to $q^{2} \in[-\infty, 0]$, and $q^{2} \in\left[0,4 m^{2}\right]$, which corresponds to the upper half circle in $x$ space.
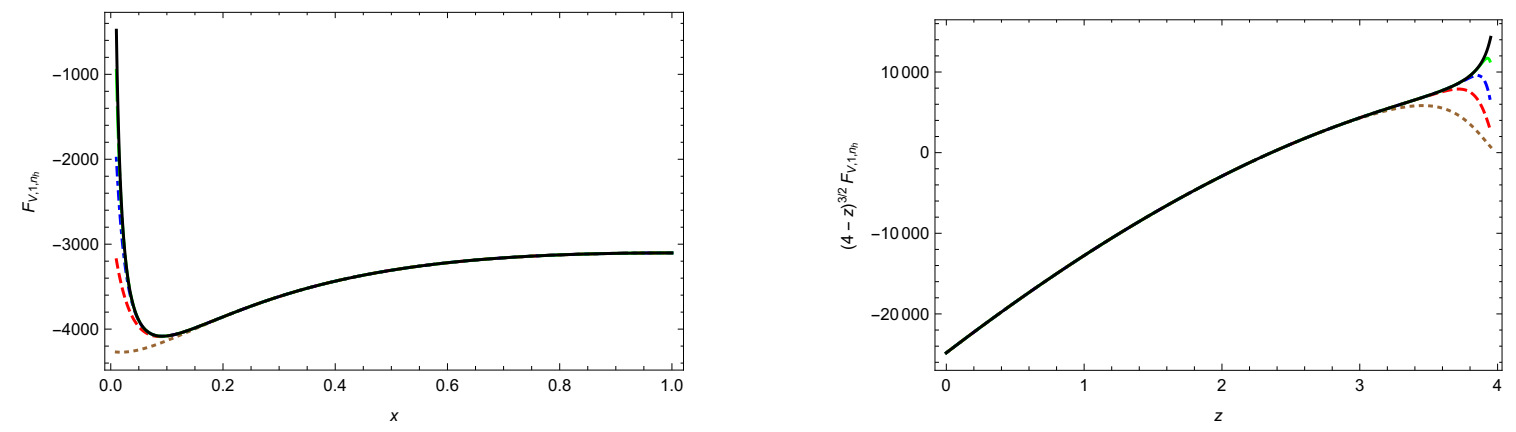

Figure 1: Vector form factor $F_{V, 1}\left(\varepsilon^{0} n_{h}^{1}\right)$, the approximation with 20, 50, 100, 200, 500 terms is shown in brown, red, blue, green and black, respectively, [1].

In Fig 2 we show again the result for the vector form factor but to better illustrate the convergences we normalize the different approximations to the most precise one.

Our results for the poles have been verified by comparing to predictions obtained from the general infrared structure of QCD amplitudes and renormalization group arguments. 


\begin{tabular}{|c|r|r|r|r|}
\hline & color & degree & order & $\begin{array}{r}\text { remaining } \\
\text { order }\end{array}$ \\
\hline$F_{V}$ & $g_{1} n_{h}$ & 1288 & 54 & 15 \\
& $g_{1} n_{h} \zeta_{3}$ & 409 & 29 & 10 \\
& $g_{1} n_{h} \zeta_{2}$ & 295 & 24 & 6 \\
& $g_{2} n_{h}$ & 1324 & 55 & 15 \\
& $g_{2} n_{h} \zeta_{3}$ & 430 & 30 & 10 \\
& $g_{2} n_{h} \zeta_{2}$ & 273 & 23 & 6 \\
\hline$F_{A}$ & $g_{1} n_{h}$ & 1314 & 54 & 15 \\
& $g_{1} n_{h} \zeta_{3}$ & 419 & 29 & 10 \\
& $g_{1} n_{h} \zeta_{2}$ & 280 & 23 & 6 \\
& $g_{2} n_{h}$ & 1130 & 52 & 15 \\
& $g_{2} n_{h} \zeta_{3}$ & 352 & 28 & 10 \\
& $g_{2} n_{h} \zeta_{2}$ & 232 & 23 & 6 \\
\hline$F_{S}$ & $n_{h}$ & 1114 & 50 & 15 \\
& $n_{h} \zeta_{3}$ & 350 & 27 & 10 \\
& $n_{h} \zeta_{2}$ & 230 & 22 & 6 \\
\hline$F_{P}$ & $n_{h}$ & 1130 & 52 & 15 \\
& $n_{h} \zeta_{3}$ & 352 & 28 & 10 \\
& $n_{h} \zeta_{2}$ & 232 & 23 & 6 \\
\hline
\end{tabular}

Table 1: Structure of the recurrences for the remaining non-first-order contributions.
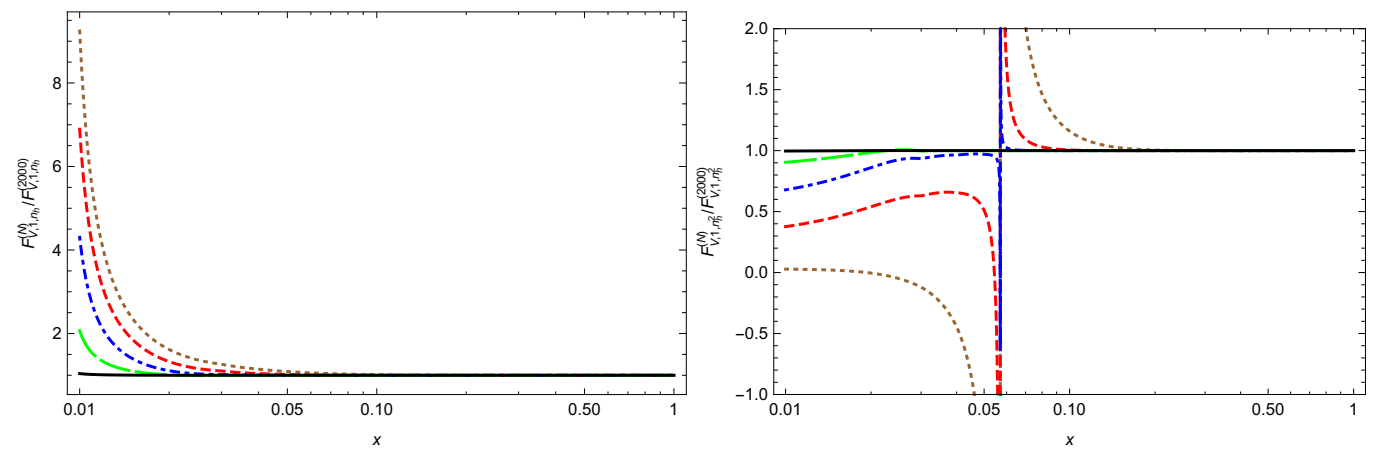

Figure 2: Ratios of the approximations with 20, 50, 100, 200, 500 terms and our best approximation using 2000 terms for the vector form factor $F_{V, 1}$. Left $\varepsilon^{0} n_{h}^{1}$, right $\varepsilon^{0} n_{h}^{2}$. The ratio using 20, 50, 100, 200, 500 terms is shown in brown, red, blue, green and black, respectively, [1].

\section{Conclusions}

We calculated the heavy-fermionic non-singlet three-loop contributions to the heavy quark form factors for vector, axial-vector, scalar and pseudoscalar currents. We applied the method of large moments to obtain deep series expansions for the final expressions, which for all pole terms and a number of finite terms could be resummed. For the remaining structures series expansions with an in priciple infinite number of terms are available. A closed analytic form for these contri- 
butions remains to be found and will be discussed in future work.

\section{Acknowledgment}

We would like to thank J. Ablinger, A. Behring, A. De Freitas, K. Schönwald and J. Vermaseren for discussions. This work was supported in part by the Austrian Science Fund (FWF) grant SFB F50 (F5009-N15), by the EU TMR network SAGEX Marie Skłodowska-Curie grant agreement No. 764850 and COST action CA16201: Unraveling new physics at the LHC through the precision frontier.

\section{References}

[1] J. Blümlein, P. Marquard, N. Rana and C. Schneider, Nucl. Phys. B 949 (2019) 114751 [arXiv:1908.00357 [hep-ph]].

[2] W. Bernreuther, R. Bonciani, T. Gehrmann, R. Heinesch, T. Leineweber, P. Mastrolia, and E. Remiddi, Nucl. Phys. B706 (2005) 245-324 [hep-ph/0406046].

[3] W. Bernreuther, R. Bonciani, T. Gehrmann, R. Heinesch, T. Leineweber, P. Mastrolia, and E. Remiddi, Nucl. Phys. B712 (2005) 229-286 [hep-ph/0412259].

[4] W. Bernreuther, R. Bonciani, T. Gehrmann, R. Heinesch, T. Leineweber, and E. Remiddi, Nucl. Phys. B723 (2005) 91-116 [hep-ph/0504190].

[5] W. Bernreuther, R. Bonciani, T. Gehrmann, R. Heinesch, P. Mastrolia, and E. Remiddi, Phys. Rev. D72 (2005) 096002 [hep-ph/0508254].

[6] J. Gluza, A. Mitov, S. Moch, and T. Riemann, JHEP 07 (2009) 001 [arXiv:0905.1137 [hep-ph]].

[7] J. Ablinger, A. Behring, J. Blümlein, G. Falcioni, A. De Freitas, P. Marquard, N. Rana and C. Schneider, Phys. Rev. D 97 (2018) no.9, 094022 [arXiv:1712.09889 [hep-ph]].

[8] J. Henn, A.V. Smirnov, V.A. Smirnov and M. Steinhauser, JHEP 1701 (2017) 074 [arXiv:1611.07535 [hep-ph]].

[9] J.M. Henn, A.V. Smirnov, and V.A. Smirnov, JHEP 12 (2016) 144 [arXiv:1611.06523 [hep-ph]].

[10] J. Ablinger, J. Blümlein, P. Marquard, N. Rana and C. Schneider, Phys. Lett. B 782 (2018) 528-532 [arXiv:1804.07313 [hep-ph]].

[11] R.N. Lee, A.V. Smirnov, V.A. Smirnov and M. Steinhauser, JHEP 03 (2018) 136 [arXiv:1801.08151 [hep-ph]].

[12] R.N. Lee, A.V. Smirnov, V.A. Smirnov and M. Steinhauser, JHEP 05 (2018) 187 [arXiv:1804.07310 [hep-ph]].

[13] J. Ablinger, J. Blümlein, P. Marquard, N. Rana and C. Schneider, Nucl. Phys. B 939 (2019) $253-291$. [arXiv:1810.12261 [hep-ph]].

[14] T. Ahmed, J.M. Henn and M. Steinhauser, JHEP 06 (2017) 125 [arXiv:1704.07846 [hep-ph]].

[15] J. Blümlein, P. Marquard and N. Rana, Phys. Rev. D 99 (2019) no.1, 016013 [arXiv:1810.08943 [hep-ph]].

[16] P. Nogueira, J. Comput. Phys. 105 (1993) 279-289. 
[17] R. Harlander, T. Seidensticker, and M. Steinhauser, Phys. Lett. B426 (1998) 125-132, [hep-ph/9712228].

[18] T. Seidensticker, in: Proc. of the 6th International Workshop on New Computing Techniques in Physics Research (AIHENP 99) Heraklion, Crete, Greece, April 12-16, 1999, hep-ph/9905298.

[19] J.A.M. Vermaseren, New features of FORM, math-ph/0010025.

[20] M. Tentyukov and J.A.M. Vermaseren, Comput. Phys. Commun. 181 (2010) 1419-1427 [hep-ph/0702279].

[21] K.G. Chetyrkin and F.V. Tkachov, Nucl. Phys. B 192 (1981) 159-204.

[22] P. Marquard and D. Seidel, The Crusher algorithm, (unpublished).

[23] J. Blümlein and C. Schneider, Phys. Lett. B 771 (2017) 31-36, [arXiv:1701.04614 [hep-ph]].

[24] M. Kauers, M. Jaroschek, and F. Johansson, in: Computer Algebra and Polynomials, Editors: J. Gutierrez, J. Schicho, Josef, M. Weimann, Lecture Notes in Computer Science 8942 (Springer, Berlin, 2015) 105-125, [arXiv:1306.4263 [cs.SC]].

[25] C. Schneider, Sém. Lothar. Combin. 56 (2007) 1-36.

[26] C. Schneider, in: Computer Algebra in Quantum Field Theory: Integration, Summation and Special Functions, Texts and Monographs in Symbolic Computation eds. C. Schneider and J. Blümlein (Springer, Wien, 2013), 325-360 [arXiv:1304.4134 [cs.SC]].

[27] C. Schneider, J. Phys. Conf. Ser. 523 (2014) 012037 [arXiv:1310.0160 [cs.SC]].

[28] J. Vermaseren, Int. J. Mod. Phys. A14 (1999) 2037-2076 [hep-ph/9806280].

[29] J. Blümlein and S. Kurth, Phys. Rev. D 60 (1999) 014018 [hep-ph/9810241].

[30] J. Ablinger, PoS (LL2014) 019 [arXiv:1407.6180[cs.SC]].

[31] J. Ablinger, A Computer Algebra Toolbox for Harmonic Sums Related to Particle Physics, Diploma Thesis, JKU Linz, 2009, arXiv:1011.1176[math-ph].

[32] J. Ablinger, Computer Algebra Algorithms for Special Functions in Particle Physics, Ph.D. Thesis, Linz U. (2012) arXiv:1305.0687[math-ph].

[33] J. Ablinger, J. Blümlein, and C. Schneider, J. Math. Phys. 52 (2011) 102301 [arXiv: 1105.6063 [math-ph]].

[34] J. Ablinger, J. Blümlein, and C. Schneider, J. Math. Phys. 54 (2013) 082301 [arXiv: 1302.0378 [math-ph]].

[35] J. Ablinger, J. Blümlein, C.G. Raab, and C. Schneider, J. Math. Phys. 55 (2014) 112301 [arXiv: 1407.1822 [hep-th]].

[36] J. Ablinger, PoS (RADCOR2017) 001 [arXiv:1801.01039 [cs.SC]].

[37] J. Blümlein and C. Schneider, Int. J. Mod. Phys. A 33 (2018) no.17, 1830015 [arXiv:1809.02889 [hep-ph]].

[38] E. Remiddi and J.A.M. Vermaseren, Int. J. Mod. Phys. A15 (2000) 725-754 [hep-ph/9905237]. 